\title{
Ansiedad matemática, actitud y autoeficacia: un estudio sobre el efecto de AppCalc en estudiantes de ingeniería
}

\author{
Mathematics anxiety, attitude and self-efficacy: \\ A study on the effect of "AppCalc" in engineering students \\ Nahina Dehesa De Gyves \\ Enrique López Ramírez
}

\begin{abstract}
RESUMEN
En este estudio se investigó la efectividad de un entorno de enseñanza en la reducción de los niveles de ansiedad en estudiantes, incremento en el sentimiento de autoeficacia y el cambio de actitudes hacia las matemáticas. Se incluyó el empleo de algunas herramientas digitales y se aplicaron tres cuestionarios involucrados en el aprendizaje de conceptos matemáticos: de ansiedad, de actitud y de autoeficacia. La investigación tuvo un diseño cuasiexperimental con un grupo que empleó la aplicación móvil $A p p C$ Calc, y un grupo control que cursó la asignatura sin el uso de $A p p C a l c$. A la par de investigar los aspectos psicosociales mencionados se indagó el efecto del empleo de la aplicación móvil sobre el manejo de ciertas habilidades básicas de matemáticas en estudiantes de ingeniería del sureste del país. Entre los hallazgos es posible mencionar que solo algunos de los resultados fueron mejores en el sentido de que pudieran ser atribuibles al empleo de la herramienta digital, sin embargo, explicitar el objetivo del estudio en el aula sí proporcionó una ventaja en la percepción docente acerca del aprendizaje del alumno.
\end{abstract}

Palabras clave: actitud, ansiedad matemática, autoeficacia, herramientas digitales.

\begin{abstract}
In this study, the effectiveness of a teaching environment in reducing anxiety levels in students, increasing the feeling of self-efficacy and changing attitudes towards mathematics was investigated. The use of some digital tools was included and three questionnaires involved in learning mathematical concepts were applied: anxiety, attitude and self-efficacy. The research had a quasi-experimental design with a group that used the mobile application $A p p C a l c$, and a control group that took the course without the use of $A p p C$ alc. Along with investigating the aforementioned psychosocial aspects, the effect of the use of the mobile application on the management of certain basic math skills in engineering students from the southeast of the country was investigated. Among the findings, it is possible to mention that only some of the results were better in the sense that they could be attributed to the use of the digital tool, however, making the aim of the study explicit in the classroom did provide an advantage in the teacher's perception of student learning.
\end{abstract}

Keywords: attitude, math anxiety, self-efficacy, digital tools. 


\section{INTRODUCCIÓN}

El aprendizaje de las matemáticas es percibido por los estudiantes de todos los niveles escolares como la asignatura más difícil en el currículo (Haase, Guimarães y Wood, 2019), y en particular se consideró durante bastantes años como un problema fundamentalmente de dimensión cognitiva (González, 2012), lo cual resulta una deducción natural si se considera que para su desarrollo intelectual se requiere de lógica, abstracción y razonamiento ordenado (George-Reyes, 2020). En contraste, en los años recientes se ha incrementado el interés de la investigación educativa por el llamado dominio afectivo en la enseñanza (Kaur y Vadhera, 2021; Goldin, 2018). Aunque ambos dominios, cognitivo y afectivo, pertenecen a la mente (Hannula, 2002), la diferencia teórica no es clara e incluso se traslapan en ocasiones (Öçal, 2021). Poder influir de manera positiva tanto en el dominio afectivo como en el cognitivo puede ser un factor motivante para realizar el estudio que a continuación se describirá.

Por una parte, la investigación en matemática educativa se enmarca en un cuerpo de conocimientos en el cual el aprendizaje de la ciencia en general en los últimos 40 años ha atravesado diversos periodos en los que definitivamente se incluye un declive en el interés de los jóvenes en continuar carreras científicas (Osborne, Simon y Collins, 2003). Si adicionalmente se considera el acelerado desarrollo de las tecnologías de información es indiscutible el cambio sustancial que han sufrido las computadoras hasta nuestros días en cómo podrían emplearse para mejorar el aprendizaje del estudiante (Clark-Wilson, Robutti y Thomas, 2020).

En tiempos actuales, la pandemia derivada de la enfermedad por coronavirus 2019 (COVID-19) ha obligado a plantearse cuestionamientos en nuevas direcciones. Si destacamos que está ampliamente aceptado que el uso de la tecnología por sí misma no es suficiente para garantizar el aprendizaje (Caseres, Pereira y Pereira, 2019) y consideramos las investigaciones que destacan al profesor como el factor más influyente en el aprendizaje del alumno (Clark-Wilson, Robutti y Thomas, 2020), entonces podría revaluarse el papel del docente con un mayor empleo de la tecnología.

Nahina Dehesa De Gyves. Profesora de tiempo completo del Tecnológico Nacional de México, campus Instituto Tecnológico del Istmo. Es doctora en Matemática Educativa con reconocimiento al Perfil PRoDeP y miembro del cuerpo de tutores de la maestría en Docencia para la Educación Media Superior (MADEMS) de la Universidad Autónoma de México. Ha participado en la elaboración de juegos de mesa en el museo de ciencias Universum. Correo electrónico: ndehesa@hotmail.com. ID: https:// orcid.org/0000-0003-2032-8639.

Enrique López Ramírez. Personal adscrito a la División de Estudios Profesionales del Tecnológico Nacional de México, campus Instituto Tecnológico de Oaxaca. Es doctor en Educación egresado de la Universidad de Guadalajara. Su línea de investigación está relacionada con actitudes, adicciones, desarrollo de aplicaciones móviles para la salud mental y para la educación. Es director de Proyectos de Investigación e Innovación en IIDTEC S.A.S. Se encuentra adscrito a la División de Posgrado e Investigación del Instituto Tecnológico de Oaxaca y también es profesor de posgrado de la Universidad Mesoamericana. Correo electrónico: quiqueohio@hotmail.com. ID: https://orcid.org/0000-0002-7526-5199. 
Anteriormente algunos investigadores ya no hablaban de tecnologías de la información y la comunicación, sino de tecnologías digitales o tecnologías portátiles (Fiallo, 2015), que ciertamente cada día son mayormente empleadas. Se ha llegado a reportar que más del 50\% de los estudiantes puede llegar a emplear el smartphone como medio para el aprendizaje de las matemáticas (George-Reyes, 2020).

Bajo el panorama mencionado, el objeto de estudio de este trabajo es analizar cómo el empleo de las tecnologías influye en las variables de ansiedad, actitud y autoeficacia en los cursos de matemáticas. Cuando en este estudio se menciona la palabra "matemáticas" específicamente se hace referencia a las áreas de álgebra y cálculo debido a que son las áreas que más han reportado elevar los niveles de ansiedad en estudiantes de nivel superior (Wu, Lin y Shih, 2016).

El miedo a los exámenes, tareas y clases de matemáticas son muy recurrentes en los estudiantes del nivel superior y tienen una característica a favor del estudio, se pueden explicar en términos de descriptores que permiten minimizar su carácter subjetivo (Eccius-Wellman y Lara-Barragán, 2016).

En primer lugar, se puede emplear al descriptor "actitudes" propuesto por Eccius-Wellman y Lara-Barragán (2016), quienes lo entienden como una expresión conductual y/o verbal que concuerda con una creencia o una emoción. También se emplean los inventarios de Tapia y Marsh (2004), que recuperan el sentir de satisfacción o rechazo al resolver un problema de matemáticas. Para el último indicador, autoeficacia, que se considera altamente asociado con la retención de estudiantes, retomamos el propuesto por Alves, Rodrígues, Rocha y Coutinho (2016) quienes se enfocan en la percepción que tienen los estudiantes para resolver problemas de ingeniería que incluyen matemáticas.

Es en la sección de instrumentos se describirán los factores y reactivos de los inventarios con mayor detalle. Cabe mencionar que el estudio se llevó a cabo con una muestra de 65 estudiantes del Tecnológico Nacional de México campus Instituto Tecnológico del Istmo, inscritos en el curso de Cálculo Vectorial del tercer semestre en las carreras de ingeniería Mecatrónica y Eléctrica. Se presenta en una primera sección del trabajo una revisión de literatura, en la que se resumen algunos factores principales que podrían influir en el aprendizaje de las matemáticas. En dicha revisión se incluyen estudios que relacionan el rendimiento escolar con factores como la actitud, la autoeficacia y la ansiedad hacia la matemática. En la sección de metodología se incluye con más precisión a los participantes y el programa de intervención. Posteriormente se presenta el análisis estadístico, para finalizar con la sección de resultados y su discusión. 


\section{MARCO TEÓRICO}

La investigación de las actitudes de los estudiantes hacia el estudio de las matemáticas ha sido motivo de trabajo de la comunidad de investigadores en educación matemática en las décadas recientes (Galende, Arrivillaga y Madariaga, 2020), y el incremento de dichos estudios se motiva por cada vez un mayor número de usuarios de las tecnologías de la información y la comunicación (TIC) (George-Reyes, 2020). En las próximas líneas analizaremos con más detalle lo que algunos autores han argumentado en torno a la forma en que las tecnologías favorecen el proceso de enseñanza-aprendizaje (Tarango, Machin-Mastromatteo y Romo-González, 2019).

Al identificar las concepciones de los docentes sobre el papel de los medios educativos en los procesos de enseñanza y aprendizaje de las matemáticas, se ha encontrado que pueden concebir los medios tecnológicos como ayudas de estudio que permiten la motivación, el apoyo a la memorización de conceptos y dan soporte para la exposición de sus temáticas (Leguizamón, Patiño y Suárez, 2015). En particular, existen estudios que destacan los componentes emocionales y actitudinales que favorecen o desfavorecen la apropiación de la tecnología como medio de aprendizaje (Orozco, García y Cepeda, 2019).

Como componente emocional se puede mencionar, por ejemplo, que las actitudes negativas hacia las matemáticas están asociadas con una mayor ansiedad (Larkin y Jorgensen, 2016) y, a su vez, una mayor ansiedad hacia las matemáticas provoca actitudes negativas hacia ellas (Rozgonjuk, Kraav, Mikkor, Orav-Puurand y Täht, 2020). $\mathrm{Y}$ esto es porque en un nivel emocional, con la ansiedad hacia las matemáticas los individuos sufren de tensión, aprensión, nerviosismo, desolación, pánico, parálisis, desorganización mental y preocupación cuando tratan de resolver un problema matemático, por lo que buscan evitar este tipo de ejercicios (Suárez-Pellicioni, Núñez-Peña y Colomé, 2016; Haase, Guimarães y Wood, 2019). Estas emociones negativas pueden bloquear la disposición, el interés y el esfuerzo de los estudiantes en la resolución de ejercicios (Kim y Hodges, 2012). Adicionalmente en el aspecto cognitivo se comprometen algunas funciones ejecutivas tales como la memoria de trabajo (Luttenberger, Wimmer y Paechter, 2018; Alves et al. 2016). Por las características mencionadas es importante determinar las fuentes de ansiedad matemática de los estudiantes y establecer un puente con el tipo de curso de ingeniería al que asisten los estudiantes. Por lo pronto, en la sección de contexto de aprendizaje se hacen explícitos algunos de los supuestos docentes que se mantuvieron implícitamente a lo largo del estudio.

Pero no solo se ha demostrado que la ansiedad se asocia con un bajo rendimiento en matemáticas (Radišiæ, Videnoviæ y Baucal, 2015; Larkin y Jorgensen, 2016; Wu, Lin y Shih, 2016; Lewis, 2019; Erturan y Jansen, 2015) al solo utilizar mecanismos de memorización sin comprender ni percibir el significado o su sentido (Tezer y Bozkurt, 2015). La confianza en sí mismo es importante en el aprendizaje de las matemáticas 
(Bartley e Ingram, 2018). Específicamente se ha demostrado que la autoeficacia percibida baja se asocia con un bajo rendimiento (Rozgonjuk et al., 2019), y viceversa (Mantooth, Usher y Love, 2020). A su vez, es considerada como un elemento que potencia la motivación y el sentimiento de logro (Star, Chen, Taylor, Durkin, Dede y Chao, 2014). Sentirse empoderado en el desempeño académico es una cualidad que Huber, Fruth, Avila-John y López-Ramirez (2016) representan con el término de autoeficacia. Y la autoeficacia en el contexto escolar es importante ya que influye en la elección, el esfuerzo, la persistencia y sentimientos de los estudiantes (Huber et al., 2016). Elevados niveles de autoeficacia hacia las matemáticas han mostrado correlación con bajos niveles de ansiedad y mejores actitudes hacia las matemáticas, por lo que reducir la ansiedad de los estudiantes por las matemáticas podría ser útil para aumentar su autoeficacia matemática (Rozgonjuk et al., 2020).

Complementariamente a lo mencionado, existen estudios que analizan la función de las tecnologías como mediadoras para superar los obstáculos relacionados con prenociones y conocimientos previos de los estudiantes (George-Reyes, 2020). Así, las TIC pueden convertirse en un recurso con el que se pueden superar obstáculos, especialmente aquellos relacionados con la prevalencia de conocimientos conceptuales y procedimentales erróneos (George-Reyes, 2020). Sin embargo, el conocimiento producido por la mediación de un artefacto nunca es independiente del artefacto (Fiallo, 2015). En un meta-análisis se reportó que cuando se han comparado los resultados de aprendizaje entre los métodos tradicionales y los métodos asistidos por tecnología, estos últimos han mostrado solo un moderado efecto positivo (Demir y Önal, 2021), por lo que se debe hacer consciente no solo de los cambios que ocurren a nivel tecnológico, sino repensar la forma de enseñar las matemáticas.

En la búsqueda de propuestas tecnológicas de corte cuantitativo de diseño cuasiexperimental aplicadas al nivel superior, se encuentra un estudio que ha tenido un efecto positivo mediante un foro virtual como estrategia de mediación sobre el aprendizaje de contenidos de cálculo diferencial (Caseres, Pereira y Pereira, 2019), en el cual se retoma como uno de los puntos favorables de su empleo la posibilidad de revisar permanentemente los conocimientos previos, siendo que contar con los conocimientos previos es requisito necesario para la conformación de nuevos aprendizajes (Castro, 2019).

\section{El contexto de aprendizaje y el uso de las TIC}

Existe una influencia de nuestra cultura y de cómo nuestra idiosincrasia permea en los hogares desde edades muy tempranas (Eccius-Wellman y Lara-Barragán, 2016); dicha repercusión también afecta el contexto escolar. Las asignaturas del nivel superior contemplan la continuación de aprendizajes matemáticos iniciados desde el nivel básico y por ello es importante atender diversas dificultades, como el empleo 
del álgebra (Flores y Azumendi 2016; Pizón y Gallardo, 2000) como respuesta a los resultados no muy alentadores de evaluaciones nacionales e internacionales (Galende, Arrivillaga y Madariaga, 2020). A favor de lo mencionado podemos argumentar que una de las ventajas de la docencia del siglo XXI formadora de estudiantes nativos digitales (Orozco, García y Cepeda, 2019) es que dichas habilidades pueden apoyar el desempeño del propio estudiante. Y esto es debido a que, en años recientes, ha existido un crecimiento exponencial en el desarrollo de aplicaciones móviles con fines instruccionales (Menekse, Anwar y Purzer, 2018).

Para retomar la continuidad del proceso de aprendizaje matemático del alumno se requiere dimensionar el curso dentro de una escala de tiempo mayor en la que ya se han venido presentando contenidos temáticos que necesitan verse aplicados a nuevos contextos (Alsina, 2009). Para contrarrestar la memorización de contenidos matemáticos por sí mismos, se requiere que el docente personifique y busque fomentar en sus alumnos una actitud específica ante las matemáticas: como una posición evaluativa desde la cual se contemplan, ordenan y categorizan los fenómenos del entorno para así poder ordenar, entender y asimilar las informaciones complejas, ambiguas e impredecibles (Galende, Arrivillaga y Madariaga, 2020).

También los profesores necesitan competencias específicas para integrar adecuadamente las herramientas tecnológicas de aprendizaje (Thurm y Barzel, 2020). A través de las tecnologías para el aprendizaje activo es posible encontrar formas de retroalimentar al estudiante que impliquen una participación más activa de su parte mediante actividades que pueden ser retomadas en lo que Rincón-Gallardo (2019) denomina "acceder a múltiples fuentes de retroalimentación que impliquen saber qué tan bien se realiza la tarea que se aprende".

Para ello, se torna prioritario enfocar la atención en el reforzamiento del rol que juega el docente en el estudiante para lograr una retroalimentación más efectiva en la que logren sentirse apoyados, ya que ello permitirá una mayor disposición y actitud hacia el uso de la herramienta tecnológica de aprendizaje (Zhai y Shi, 2020). Para esta mediación se consideró desde el inicio del presente estudio que la retroalimentación inmediata es fundamental (Yeh, Cheng, Chen, Liao y Chan, 2019).

$\mathrm{Al}$ respecto la tecnología educativa tiene mucho que decir, ya que puede estructurar un entorno, al que llamamos "entorno de enseñanza", donde se pretende que sea posible el reforzamiento de las intervenciones basadas en la evidencia empírica en secuencias que pudieran permitir tener mayor control con el entorno y las actividades (Chung, 2019). Siendo que el aprendizaje activo reduce la ansiedad (Rozgonjuk et al., 2019) y permite encontrar sentido y significado a lo que se aprende, en nuestra retroalimentación se pretendió llevar desde el inicio de la intervención la plataforma Classroom (en su formato de foro), la cual debería permitir una comunicación más fluida con respecto al tiempo real. 
También la tecnología ha tenido una extraordinaria capacidad para registrar, obtener, almacenar y operar con datos discretos sobre la actividad de personas (Castañeda, 2019), y aunque no se controló un entorno en el que se pudiera videograbar al alumno para posteriormente ser analizado (Chung, 2019), sí se consideró que el empleo de teléfonos inteligentes pudiera mediar el objetivo docente. Los teléfonos inteligentes son objeto de desarrollo de contenidos educativos digitales que buscan incorporar elementos audiovisuales para ser más atractivos al ser consultados (Domínguez, Organista y López, 2018). Y al no contar con un equipo de diseño instruccional que procesara un análisis, diseño, desarrollo, implementación y evaluación de un recurso educativo (Domínguez, Organista y López, 2018), en nuestro estudio empleamos AppCalc, desarrollado por estudiantes del TecNM. En el periodo escolar II-2018 un equipo de estudiantes del tercer semestre de Ingeniería en Sistemas Computacionales y Mecatrónica desarrolló una app para Android que funcionó en cualquier tipo de dispositivo móvil a partir de la versión 4.4.4. La aplicación para celular $A p p C a l c$ muestra un primer menú como se muestra en las figuras 1 y 2.
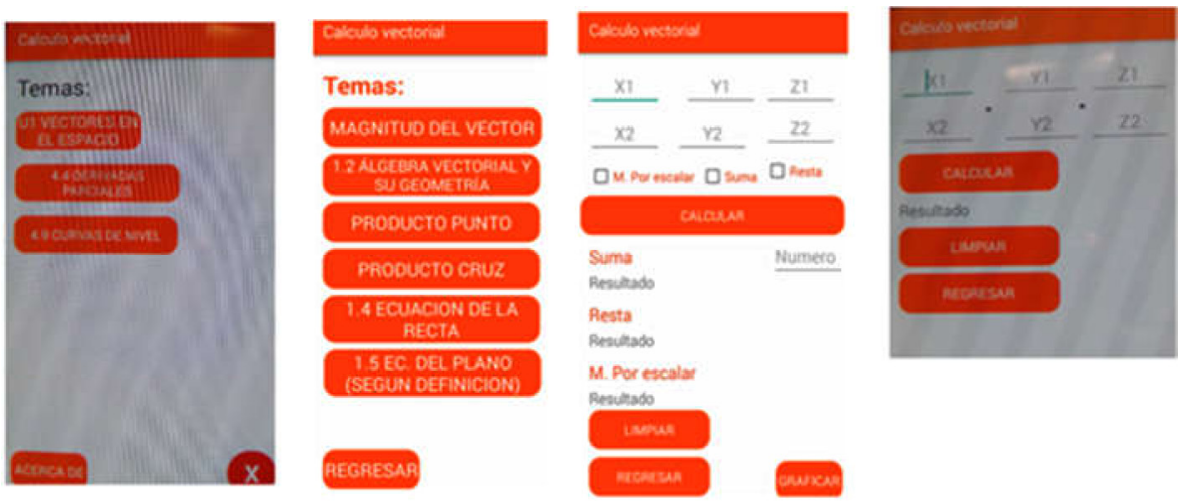

Figura 1. Menú principal, temas de la unidad I, operaciones con vectores y producto punto.
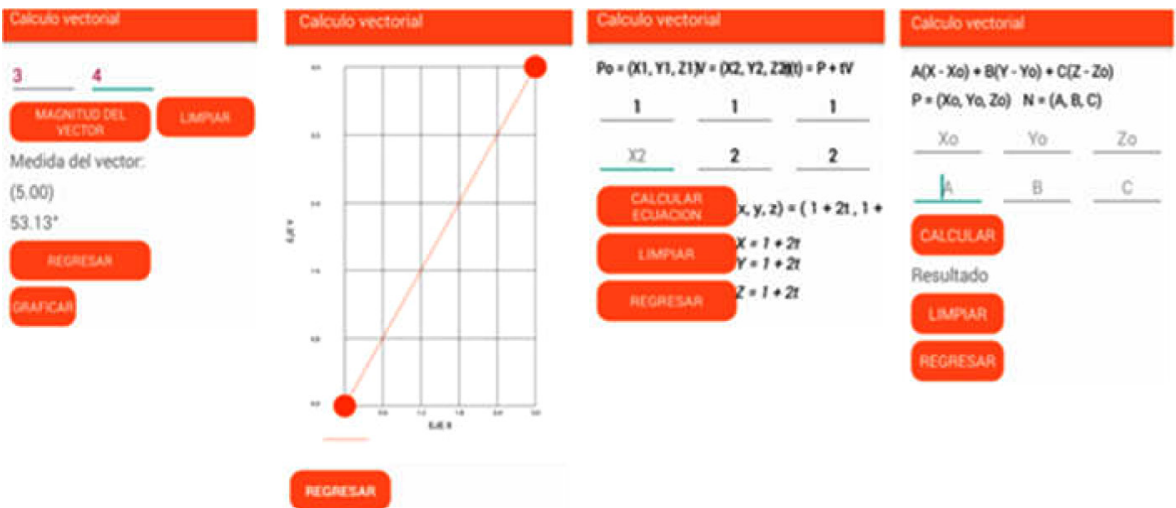

Figura 2. Módulo y argumento de un vector, ecuación de la recta y ecuación del plano. Desarrolladores: Juan Raúl Martínez López, Miguel Ángel Toledo Santiago, estudiantes de tercer semestre de Ingeniería en Sistemas Computacionales, y Rey Iván García Domínguez, de Ingeniería Mecatrónica. 


\section{Planteamiento del problema}

Aplicar la matemática aún en sus conocimientos básicos de aritmética y álgebra no es tarea fácil en cualquier nivel, incluyendo el nivel superior. En este trabajo, más que solo centrarse en la matemática del nivel superior, se propuso un entorno en el que se pudieran integrar aspectos psicosociales como una mejor actitud hacia la matemática. Para ello el docente hace uso de un entorno virtual, que por un lado le permitiera tener un mayor registro para dar seguimiento al diálogo con su clase y por otro lado, y más importante, que el alumno pudiera aumentar la sensación de empoderamiento al tener más recursos para controlar y para expresarse. La problemática entonces cuestiona si la aplicación móvil puede lograr reducir la ansiedad en el aprendizaje y provocar una mejoría en cuanto a la actitud y la autoeficacia matemática.

\section{MÉtodo}

En el semestre agosto-diciembre 2019 se impartió el curso de Cálculo Vectorial a dos grupos denominados experimental y control, en el Tecnológico Nacional campus Instituto Tecnológico del Istmo.

El grupo experimental, a diferencia del grupo control (quienes solo recibían las clases expositivas), a la par de las clases presenciales se les invitaba a visitar una plataforma de Classroom en la que podían acceder al material audiovisual (información teórica en formato pdf, video-tutoriales, etc.) correspondiente a los temas vistos en clases. La plataforma fungió como un foro en el que podían opinar y descargar desde el inicio del curso una aplicación para celular denominada $A p p C a l c$.

En cuanto a la justificación de los temas vistos en función de las dificultades de aprendizaje reportadas en la bibliografía, iniciamos con el programa de estudios de la asignatura de Cálculo Vectorial, el cual incluye la obtención de funciones paramétricas de la recta, el plano, álgebra vectorial, plano polar, integración y derivación de funciones de varias variables, por mencionar algunos temas. Se puede observar que en el curso de Cálculo Vectorial que nos ocupa se requiere no solo de la graficación de vectores y funciones en el plano cartesiano, también del manejo de operaciones numéricas y del teorema de Pitágoras, por mencionar algunos ejemplos. La misma elaboración de tablas para el cálculo de puntos de la función en cuestión también requiere de numerosos cálculos, los cuales las calculadoras pueden acortar los tiempos para realizar. En la figura 3 se ejemplifica cómo un estudiante practica el concepto básico de función cuadrática con la aplicación $A p p C$ Calc.

Sin lugar a dudas, la aritmética, el álgebra, la geometría y la trigonometría son prerrequisitos para desarrollar los temas del curso de Cálculo Vectorial. Sin embargo, si nuestro entorno cultural tiende a verlas de forma mecánica y memorística (Álvarez, González, Lampón, Otero y Zorrilla, 2008), eso explicaría en gran parte por qué 


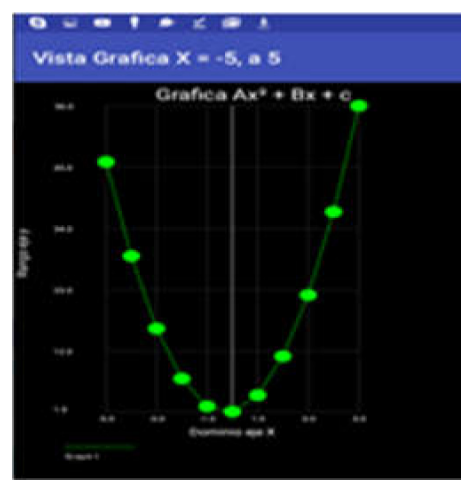

Figura 3. Se requiere que el alumno tabule puntos de una función cuadrática vista en cursos anteriores.

constatamos con demasiada frecuencia la falta de dominio de ellas en el nivel superior. Siguiendo la recomendación de Álvarez et al. (2008) de incluir a la par una sección de contextualización y problematización de los contenidos, a lo que denomina "pasar de un nivel 1 de contenidos a un nivel 2 de habilidades", el curso de Cálculo Vectorial se presenta como una excelente oportunidad para seguir integrando los conocimientos básicos de aritmética y álgebra.

Y precisamente en dicha transición de niveles, la participación y validación del docente es esencial, al apoyar a identificar el problema, los datos relevantes y los irrelevantes, interpretar qué hacer con los datos, conformar un plan de solución. Soto, Álvarez, Otero, Lampón y Grinda (2006) lo resumen de la siguiente manera: "Comenzar a hacer conexiones e integración de situaciones para solucionar un problema usando matemáticas”. Es por ello que es pertinente cuestionarse si la aplicación móvil contribuye a favorecer dicha integración.

\section{El programa de intervención}

Una vez conformados los dos grupos, experimental y control, se llevó a cabo el estudio cuasiexperimental para conocer los efectos de la aplicación móvil sobre las actitudes hacia las matemáticas, ansiedad hacia las matemáticas, el sentido de autoeficacia con respecto a las matemáticas y las calificaciones obtenidas en el examen después de la intervención, tal como se muestra en la tabla 1. Cada test tuvo una duración máxima de 15 minutos y el examen de matemáticas fue resuelto aproximadamente en 40 minutos.

Tabla 1. Fecha de aplicación de reactivo.

Temas

Aplicación de encuestas y examen de Matemática básica

Unidad I: Graficación de vectores, módulo de vectores. Producto punto y producto cruz, Ecuación de la recta y Ecuación del plano

Aplicación de pretest: 3 al 7 septiembre del 2018

Otros temas que no se vieron con AppCalc

Lapso sin aplicar tests

Unidad V: Derivadas parciales y Curvas de nivel

Aplicación de postest: 19 al 23 noviembre del 2018 


\section{Selección de la muestra}

La investigación se llevó a cabo con estudiantes de ingeniería en Mecatrónica y Eléctrica inscritos en el tercer semestre en el Instituto Tecnológico del Istmo. La muestra estuvo conformada por 30 estudiantes del grupo control y 35 estudiantes del grupo experimental.

\section{Inventario de actitudes hacia las matemáticas (Tapia y Marsh, 2004)}

Se aplicó el cuestionario "Inventario de actitudes hacia las matemáticas", desarrollado por Tapia y Marsh (2004). El cuestionario se compone de 40 reactivos agrupados en cuatro subescalas y se mide a través de la escala de Likert que va desde en total desacuerdo hasta fuertemente de acuerdo. Puntajes altos en el cuestionario indican mejores actitudes hacia las matemáticas. El cuestionario tiene cuatro dimensiones: 1) autoconfianza (15 reactivos), 2) valor sobre las matemáticas (10 reactivos), 3) gozo (10 reactivos) y 4) motivación (5 reactivos). En su validación original se obtuvo un índice de consistencia interna de 0.96. La versión en español que se utilizó para el presente estudio fue traducida por Guevara (2008). El cuestionario se compone de preguntas como las siguientes: 1) Las matemáticas son una materia necesaria y de mucho valor, 2) Me siento muy satisfecho cuando resuelvo un problema de matemáticas.

\section{Cuestionario sobre ansiedad matemática (Eccius-Wellmann, Lara- Barragan, 2016)}

El cuestionario consta de 30 reactivos, se mide a través de una escala de Likert, la alternativa de respuesta es casi nunca, a veces, más o menos la mitad de las veces, con frecuencia, casi siempre. Se compone de tres factores: actitudes, creencias y emociones.

En la validación original del instrumento se obtuvo el .91 de alfa de Cronbach.

\section{Inventario de autoeficacia hacia las matemáticas (Alves et al., 2016)}

Es un cuestionario construido para evaluar la autoeficacia en estudiantes de ingeniería. Se compone de 12 reactivos medidos a través de una escala de Likert. Los autores consideraron que es el cuestionario que mejor se adaptaría para cumplir con los objetivos del estudio, por lo que se realizó una traducción ad hoc del inglés al español a través del procedimiento traducción directa-traducción inversa y entrevistas con otros profesores del área de Matemáticas para corroborar que los reactivos serían comprendidos por los estudiantes.

De los tres factores del instrumento, únicamente se tomaron los 12 reactivos del factor autoeficacia, que en el análisis de fiabilidad obtuvo un índice de consistencia interna de .80 con el $32.33 \%$ de la varianza explicada. 


\section{Conocimientos matemáticos}

Para la selección del cuestionario que diera cuenta de la evaluación de conocimientos básicos de matemáticas se atendió a la recomendación de no solo emplear reactivos de mecanización y memorización de algoritmos sino incluir una sección de contextualización y problematización de los contenidos en lo que se denominó "pasar de un nivel 1 de contenidos a un nivel 2 de habilidades" (Álvarez et al., 2008). Para comenzar a hacer conexiones e integración de situaciones para solucionar un problema usando matemáticas (Soto et al., 2006), el docente se propuso en sus clases identificar el problema, los datos relevantes y los irrelevantes, interpretar qué hacer con los datos y conformar un plan de solución.

Se emplearon 10 reactivos divididos en las dos secciones de conocimientos y de habilidades, propuestas por Álvarez et al. (2008) y Soto et al. (2006) respectivamente. En la sección 1 se hace uso de procedimientos y algoritmos estándares en los que se emplean habilidades técnicas, expresiones simbólicas y fórmulas ya empleadas en niveles escolares anteriores. En la sección 2 se hace referencia a descifrar e interpretar lenguaje simbólico y formal, y entender sus relaciones con el lenguaje natural, manejar las proposiciones y expresiones que contienen símbolos y fórmulas, usar variables, solucionar ecuaciones y ejecutar cálculos.

\section{Análisis estadístico}

Para conocer las diferencias antes y después de la aplicación de la estrategia de aprendizaje a través de la aplicación móvil, se ejecutaron pruebas $t$ de Student para muestras relacionadas a través del programa SPSS versión 27. Se realizaron análisis entre grupos y dentro de los grupos para ambos grupos, GE y GC.

\section{Resultados}

\section{Ansiedad}

Podemos observar en la tabla 2 que en lo que respecta a las mediciones correspondientes a las variables actitudes hacia las matemáticas, creencias y emociones, el grupo

Tabla 2. Resultados en la variable ansiedad hacia las matemáticas.

\begin{tabular}{lcccc}
\multicolumn{1}{c}{ Variables } & $\begin{array}{c}\text { GE pretest } \\
\text { M (DE) }\end{array}$ & $\begin{array}{c}\text { GE postest } \\
\text { M(DE) }\end{array}$ & GC pretest & GC postest \\
\hline Actitudes & $14.72(4.01)$ & $15.24(4.39)$ & $15.95(5.14)$ & $17.87(4.32)^{*}$ \\
\hline Creencias & $71.20(15.14)$ & $75.60(18.32)$ & $76.29(21.18)$ & $89.04(17.84)^{*}$ \\
\hline Emociones & $11.16(3.47)$ & $11.92(3.31)$ & $11.58(3.82)$ & $14.70(4.12)^{*}$ \\
\hline Ansiedad total & $45.32(9.59)$ & $48.44(12.09)$ & $48.75(/ 14.20)$ & $56.45(11.18)$ \\
\hline
\end{tabular}

M: Media, DE: Desviación estándar, GE: Grupo experimental, GC: Grupo control.

Fuente: Elaboración propia. 
experimental no mostró cambios significativos de la medición pretest a la medición postest, sin embargo, el grupo control sí mostró un cambio estadísticamente significativo para las mismas variables. Por otra parte, al sumar todas las variables para considerarlas como una medición de ansiedad global, ambos grupos no reflejaron cambios estadísticamente significativos.

A partir de lo mostrado en la tabla 2 (para GE 45.32 [9.59] versus 48.44 [12.09] y para GC 48.75[/14.20] versus 56.45 [11.18]), podemos concluir que las pruebas de Eccius-Wellmann y Lara-Barragán no mostraron diferencias significativas en la ansiedad hacia las matemáticas entre estudiantes que emplearon $A p p C a l c$. A pesar de que los dos grupos mostraron una mejoría con respecto al inicio de la intervención, lo cierto es que los datos reflejan que la herramienta digital no hace una diferencia sustancial, aun cuando la percepción del docente es que la tecnología empodera al estudiante, de tal forma que le permite manejar de mejor manera su ansiedad.

\section{Actitudes}

Podemos apreciar en la tabla 3 las variables correspondientes al componente actitudinal de nuestro estudio. De manera general, se distingue que tanto el grupo control como el grupo experimental muestran diferencias positivas respecto al inicio de la intervención, en especial para las variables autoconfianza y motivación, ambos grupos mostraron cambios significativos antes y después de la intervención, sin embargo, en las variables valor sobre las matemáticas y gozo, solo el grupo experimental reflejó cambios estadísticamente significativos.

Tabla 3. Resultados en la variable actitud hacia las matemáticas.

\begin{tabular}{|c|c|c|c|c|}
\hline Variables & $\begin{array}{c}\text { GE pretest } \\
\mathrm{M}(\mathrm{DE})\end{array}$ & $\begin{array}{c}\text { GE postest } \\
\text { M(DE) }\end{array}$ & GC pretest & GC postest \\
\hline Autoconfianza & $38.56(6.98)$ & $55.52(2.02)^{*}$ & $43.16(7.51)$ & $48.22(12.31)^{*}$ \\
\hline Valor sobre las matemáticas & $37.48(11.47)$ & $44.68(8.04)^{*}$ & $39.00(7.25)$ & $39.29(9.14)$ \\
\hline Gozo & $22.88(3.19)$ & $31.20(2.27)^{*}$ & $27.16(3.76)$ & $27.95(6.90)$ \\
\hline Motivación & $13.24(2.27)$ & $31.20(3.95)^{* * *}$ & $16.41(3.69)$ & $17.95(5.78) *$ \\
\hline Actitud global (3) & $226.56(33.66)$ & $300.96(48.86)^{*}$ & $255.87(29.42)$ & $263.79(58.38)^{*}$ \\
\hline
\end{tabular}

M: Media, DE: Desviación estándar, GE: Grupo experimental, GC: Grupo control.

Fuente: Elaboración propia.

Es decir que, aunque los dos grupos muestran una mejoría, es interesante notar el valor añadido del grupo experimental en las variables de gozo y valoración de las matemáticas correspondiente al grupo que empleó la herramienta digital. 


\section{Autoeficacia hacia las matemáticas}

Con respecto al cuestionario autoeficacia hacia las matemáticas, cuyos reactivos corresponden a un solo factor, podemos ver la tabla 4. Se encontró que únicamente los estudiantes del grupo control reflejaron cambios estadísticamente significativos en las mediciones postest.

Tabla 4. Resultados en la variable autoeficacia hacia las matemáticas.

\begin{tabular}{ccccc} 
Ge pretest & GE postest & & \\
Variables & $\mathrm{M}(\mathrm{DE})$ & $\mathrm{M}(\mathrm{DE})$ & GC pretest & GC postest \\
\hline Autoeficacia & $32.62(7.12)$ & $34.62(5.64)$ & $34.72(4.41)$ & $41.96(6.52)^{*}$ \\
\hline
\end{tabular}

M: Media, DE: Desviación estándar, GE: Grupo experimental, GC: Grupo control.

Fuente: Elaboración propia.

\section{Conocimientos básicos}

En cuanto a la evaluación de los conocimientos básicos de la matemática, podemos observar la tabla 5 .

En ella podemos observar que el grupo control obtuvo un promedio ligeramente mayor al grupo experimental en el inicio de la intervención de este estudio. Sin embargo, en la aplicación del postest, observamos que los dos grupos tuvieron mejoría y aunque ni uno de los dos grupos tuvo un promedio aprobatorio; el grupo experimental sí tuvo mejoría con respecto al grupo control. La ligera mejoría (aunque no aprobatoria) del postest con respecto al pretest de los dos grupos cobra relevancia respecto a lo que se fomentó: recordar conceptos de forma memorística complementariamente a realizar nuevas conexiones al aplicar la matemática a problemas más cercanos a la ingeniería.

Con la finalidad de ahondar más en este punto, recordemos que los aspectos que se abordan en la parte de evaluación matemática son conceptos básicos (no los de Cálculo Vectorial) que se mantienen como no aprobatorias en las dos evaluaciones y,

Tabla 5. Conocimientos básicos de matemáticas.

\begin{tabular}{|c|c|c|c|c|}
\hline & $\begin{array}{c}\text { GE pretest } \\
\text { (promedio grupal) }\end{array}$ & $\begin{array}{c}\text { GE postest } \\
\text { (promedio grupal) }\end{array}$ & $\begin{array}{c}\text { GC pretest } \\
\text { (promedio grupal) }\end{array}$ & $\begin{array}{c}\text { GC postest } \\
\text { (promedio grupal) }\end{array}$ \\
\hline Variables & M & M & M & M \\
\hline Conocimientos básicos & 12.82 & 46.52 & 17.29 & 45.00 \\
\hline \multicolumn{5}{|c|}{ Análisis intra- grupo } \\
\hline & GC pretest & GC postest & & \\
\hline Variables & $\mathrm{M}(\mathrm{DE})$ & $\mathrm{M}(\mathrm{DE})$ & GE pretest & GC postest \\
\hline Calificaciones & $1.73(1.68)$ & $4.5(1.99)^{*}$ & $1.38(1.68)$ & $5.42(4.77)^{*}$ \\
\hline
\end{tabular}

M: Media, DE: Desviación estándar, GE: Grupo experimental, GC: Grupo control.

Fuente: Elaboración propia. 
más que corroborar lo mal del sistema educativo, lo anterior muestra un síntoma en cuanto que los reactivos pueden precisarse más. Aunque la investigación se enfocó en mayor medida a aspectos psicosociales, el instrumento de evaluación matemática por sí mismo fue insuficiente y debe estudiarse más (por ejemplo con entrevistas).

\section{Análisis entre los grupos}

En la tabla 6 se expone la comparación de las tablas anteriores. En ella se resume que no se encontraron diferencias significativas en el análisis entre grupos. Se realizaron dos comparaciones entre ambos, primero en la evaluación pretest, en la que no se reflejaron diferencias significativas $(t=.51)$, por lo que pudimos partir de la igualdad de condiciones en cuanto a las calificaciones antes de comenzar con el curso. En la segunda evaluación se compararon nuevamente las calificaciones de ambos grupos, y tampoco se reflejaron diferencias significativas $(t=-.00)$.

Se encontraron diferencias significativas en las actitudes en la puntuación pretest en los grupos GE y GC a favor del GC ( $t=3.04)$. En las puntuaciones postest de autoeficacia en las comparaciones entre grupos también existieron diferencias significativas, obteniendo mejores resultados el grupo experimental.

Tabla 6. Análisis entre los grupos.

\begin{tabular}{|c|c|c|c|c|}
\hline Variables & $\begin{array}{c}\text { GEPR } \\
\text { M (DE) }\end{array}$ & $\begin{array}{l}\text { GCPRE } \\
\text { M (DE) }\end{array}$ & $\begin{array}{l}\text { GE POS } \\
\text { M (DE) }\end{array}$ & $\begin{array}{l}\text { GC POS } \\
\text { M (DE) }\end{array}$ \\
\hline Actitudes & 226.56 & $255.87(29.42)$ & $300.96(48.86)^{*}$ & $263.79(58.38)^{*}$ \\
\hline total & $(33.66)$ & 3.04 & & \\
\hline Ansiedad & $45.32(9.59)$ & $48.75(/ 14.20)$ & $48.44(12.09)$ & $56.45(11.18)$ \\
\hline \multicolumn{5}{|l|}{ total } \\
\hline Autoeficacia & $33.62(7.12)$ & $34.72(4.41)$ & $34.62(5.64)$ & $41.96(6.52)^{*}$ \\
\hline
\end{tabular}

Fuente: Elaboración propia.

Tabla 7. Análisis entre grupos de las calificaciones.

\begin{tabular}{lcccc} 
& GE pretest & GE postest & & \\
Variables & M (DE) & M(DE) & GC pretest & GE postest \\
\hline Calificaciones & $1.72(1.68)$ & $1.43(1.69)$ & $4.50(1.99)$ \\
\hline
\end{tabular}




\section{Discusión DE RESUltados}

El propósito principal de este trabajo fue probar la efectividad de un entorno de enseñanza en la que se incluyera el empleo de algunas herramientas digitales, en particular $A p p C a l c$. Nos referimos al entorno de enseñanza, no solo a una clase que emplea una $a p p$, sino a un entorno en el que la app permite una retroalimentación activa con el estudiante. Es decir, el supuesto subyacente es que manejar manualmente los temas mediante la app permite en paralelo el dominio de su propia ansiedad, actitud y autoeficacia hacia las matemáticas. En paralelo también involucra que las matemáticas manejadas por la app permiten estar al alcance de sus manos en lugar de solo ser vistas de forma lejana y abstracta.

Existen estudios que han reportado que los estudiantes atribuyen su bajo rendimiento principalmente a factores que están fuera de su control, tales como la calidad de la instrucción recibida o el estilo de enseñanza del profesorado (Pyzdrowski, Sun, Curtis, Miller, Winn y Hensel, 2013). Para contrarrestar lo anterior se retomaron sugerencias generales como que los profesores refuercen la motivación de los estudiantes a través de propiciar la búsqueda de asesorías extra clase o de oficina (Pyzdrowski et al., 2013); que el material que se utilice en los medios tecnológicos sea interesante, importante y sobre todo que tenga el sentido de utilidad para lograr que los estudiantes se involucren, y reforzar el conocimiento previo de álgebra y trigonometría (Pyzdrowski et al., 2013).

En cuanto a las medidas particulares, no bastó la recomendación verbal de que con AppCalc se pretendía ejercitar herramientas digitales para resolver problemas propios de la ingeniería en un escenario futuro en el que la teoría matemática se demandará en un contexto más utilitario. En los resultados del cuestionario de autoeficacia no se evidenció el beneficio de haber empleado dicha "operatividad tecnológica" al resolver los problemas matemáticos como antesala a su actuar profesional como futuros ingenieros. Podemos atribuir estos resultados a que el tiempo de entrenamiento y evaluación fue muy corto, por lo que consideramos importante en escenarios futuros ampliar el tiempo, posiblemente desde los temas más básicos hasta los más complejos con el mismo grupo.

Aunque el cuestionario de autoeficacia pretendió medir y proporcionar información a los investigadores con respecto a si se puede extrapolar la utilidad matemática en contextos casi similares a los que se enfrentarán como futuros ingenieros, nuestro enfoque fue más ambicioso al evaluar y fomentar la habilidad de resolver problemas matemáticos y no nos limitamos solo a evaluar el conocimiento memorístico a corto plazo. Sin embargo, los resultados del examen de conocimientos básicos de matemáticas revelados en este estudio evidencian que, aunque mejoraron, la calificación no fue aprobatoria de acuerdo al reglamento de la institución, no obstante, consideramos que, debido a lo complejo que se puede volver el aprendizaje del cálculo, obtener 
una mejoría, aunque no significativa en términos estadísticos, podría considerarse ventajoso.

La afirmación de la importancia de que los dos grupos presentaran una ligera mejoría con datos estadísticamente significativos para el grupo control en actitud y autoeficacia y para ambos grupos en las calificaciones puede ser modesta, pero para este trabajo estos cambios muestran evidencia de que se requiere ampliar el periodo de evaluación a más de un semestre para medir adecuadamente el logro de las competencias. Independientemente de los resultados, las herramientas didácticas de la aplicación sí se sumaron a la meta de proporcionar "oportunidades múltiples de retroalimentación".

En resumen, de los cuestionarios aplicados, solo en el de actitudes se observó una diferencia significativa atribuible a la aplicación de $A p p$ Calc, lo cual nos llevaría a repensar algunos supuestos docentes que, aunque fueron inspiradores, requieren de precisarlos de la siguiente manera: "con una herramienta digital, el estudiante puede empoderarse y con ello podría disminuir sus niveles de ansiedad". Estadísticamente no se pudo sostener dicha afirmación y deja abierta la discusión en la consideración de otros factores a ser contemplados en futuros escenarios y estudios. Además, la posibilidad de que el empleo de una herramienta digital pudiera contribuir a la autoimagen del estudiante al acercarse a una naturaleza "más" digital no pudo clarificarse estadísticamente.

Por las características del estudio, no se incluyó a la ansiedad ni a la actitud docente, a pesar de la relación directa que tienen dichos factores en la toma decisiones al interior del aula, sin embargo, para futuros estudios incluiremos a los docentes como sujetos de estudio. Un ejemplo de ansiedad docente fue observar que el grupo control tuvo desde el inicio del estudio un puntaje ligeramente mayor en comparación con el grupo experimental. Aceptar las condiciones cotidianas es, desde una perspectiva docente, de mayor aplicabilidad, y si se cuenta con herramientas estadísticas se pueden encontrar resultados que muy difícilmente se pudieran observar sin el apoyo estadístico. Es decir, como docentes, no siempre se pueden observar pequeños cambios actitudinales debido a que su medición requiere de cierto rigor no necesariamente compatible con la disponibilidad del docente. Si adicionalmente la observación es grupal, sin duda el apoyo estadístico tiene un alcance mayor. En este sentido, confirmar la hipótesis de que $A p p C a l c$ sí presentó una diferencia significativa en cuanto a la actitud del estudiante, es un resultado que impacta y corrobora la percepción del docente de manera favorable.

Dilucidar la diferencia teórica que todavía existe entre la ansiedad hacia las matemáticas y la ansiedad hacia los exámenes de matemáticas (Erturan y Jansen, 2015) es una recomendación a considerar, conveniente para próximos estudios. Tal como ya se mencionó, ello podría explicar en mayor medida qué tanto influye el instrumento en la valoración del docente en el desempeño del estudiante. 


\section{REFERENCIAS}

Alsina, Á. (2009). El aprendizaje realista: una contribución de la investigación en educación matemática a la formación del profesorado. En M. J. González, M. T. González y J. Murillo (eds.), Investigación en educación matemática XIII (pp. 119-127). Santander: SEIEM.

Álvarez, A., González, M., Lampón, J., Otero, G., y Zorrilla, J. (2008). Desarrollo de habilidades verbales y matemáticas II. México: AGO Editorial.

Alves, M., Rodrígues, C., Rocha, A., y Coutinho, C. (2016). Self-efficacy, mathematics' anxiety and perceived importance: An empirical study with Portuguese engineering students. European Journal of Engineering Education, 41(1), 105-121, DOI: 10.1080/03043797.2015.1095159.

Bartley, S. R., e Ingram, N. (2018). Parental modelling of mathematical affect: Self-efficacy and emotional arousal. Mathematics Education Research Journal, 30(3), $277-$ 297. https://doi.org/10.1007/s13394-017-0233-3.

Caseres, E. A., Pereira, Z., y Pereira, L. C. (2019). Efecto del foro virtual sobre el aprendizaje de cálculo diferencial. Revista Electrónica de Investigación Educativa, 21(1), 1. https://doi.org/10.24320/redie.2019.21.e30.2051.

Castañeda, L. (2019). Debates sobre tecnología y educación: caminos contemporáneos y conversaciones pendientes. RIED. Revista Iberoamericana de Educación a Distancia, 22(1). https://doi.org/10.5944/ried.22.1.23020.

Castro, W. O. (2019). La complejidad paradigmática en el aprendizaje significativo de las matemáticas. Revista Educare-UPEL-IPB-Segunda Nueva Etapa 2.0, 23(2), 77-91.

Demir, C. G., y Önal, N. (2021). The effect of technologyassisted and project-based learning approaches on students' attitudes towards mathematics and their academic achievement. Education and Information Technologies, 26(3), 3375-3397.

Chung, E. Y. H. (2018). Robotic intervention program for enhancement of social engagement among children with autism spectrum disorder. Journal of Developmental and Physical Disabilities, 31(4), 419-434. doi: 10.1007/ s10882-018-9651-8.

Clark-Wilson, A., Robutti, O., y Thomas, M. (2020). Teaching with digital technology. ZDM, 52(7), 1223-1242. https://doi.org/10.1007/s11858-020-01196-0.
Domínguez Pérez, C., Organista Sandoval, J., y López Ornelas, M. (2018). Diseño instruccional para el desarrollo de contenidos educativos digitales para teléfonos inteligentes. Apertura, 10(2), 80-93.

Eccius-Wellmann, C., y Lara-Barragán Gómez, A. (2016). Hacia un perfil de ansiedad matemática en estudiantes de nivel superior. Revista Iberoamericana de Educación Superior, 7(18), 109-129.

Erturan, S., y Jansen, B. (2015). An investigation of boys' and girls' emotional experience of math, their math performance, and the relation between these variables. European Journal of Psychology of Education, 30(4), 421-435. https://doi.org/10.1007/s10212015-0248-7.

Fiallo, J. (2015). Acerca de la investigación en educación matemática desde las tecnologías de la información y la comunicación. Actualidades Pedagógicas, (66), 69-83. doi: https://doi.org/10.19052/ap.3436.

Flores, W. O., y Auzmendi, E. (2016). Los problemas de comprensión del álgebra en estudiantes universitarios. Ciencia e Interculturalidad, 19(2), 54-64. DOI: http://dx.doi.org/10.5377/rci.v19i2.3119.

Fuentes-Guevara, M. G. (2008). Variables actitudinales y motivacionales relacionadas con el logro matemático en la Universidad de las Américas-Puebla [Tesis de Maestría]. Universidad de las Américas, Puebla. http://repositorio.udlap.mx/xmlui/handle/123456789/8015.

Galende, N., Arrivillaga, A. R., y Madariaga, J. M. (2020). Attitudes towards mathematics in secondary school students. Personal and family factors. Culture and Education, 32(3), 529555, DOI: $10.1080 / 11356405.2020 .1785156$.

George-Reyes, C. E. (2020). Reducción de obstáculos de aprendizaje en matemáticas con el uso de las TIC. IE Revista de Investigación Educativa de la REDIECH, 11, [e697]. https://doi.org/10.33010/ie_rie_rediech. v11i0.697.

Goldin, G. A. (2018). Discrete mathematics and the affective dimension of mathematical learning and engagement. En Teaching and learning discrete mathematics worldwide: Curriculum and research (pp. 53-65). Cham: Springer. DOI: 10.1007/978-3-319-70308-4. 
González, R. (2012). Cambio de actitudes y creencias hacia las matemáticas. Intervención con perspectiva de género en escuelas secundarias. México: UPN.

Haase, V. G., Guimarães, A. P. L., y Wood, G. (2019). Mathematics and emotions: The case of math anxiety. En International handbook of mathematical learning difficulties (pp. 469-503). Cham: Springer.

Hannula, M. S. (2002). Attitude towards mathematics: Emotions, expectations and values. Educational studies in Mathematics, 49(1), 25-46. DOI: 10.1023/A: 1016048823497.

Huber, M., Fruth, J. D., Avila-John, A., y López-Ramirez, E. (2016). Teacher self-efficacy and student outcomes: A transactional approach to prevention. Journal of Education and Human Development, 5(1), 46-54. https: / / doi.org/10.15640/jehd.v5n1a5.

Kaur, N., y Vadhera, R. P. (2021). Predicting students' achievement in science from selected affective factors. SN Social Sciences, 1(1), 1-19.

Kim, C., y Hodges, C. B. (2012). Effects of an emotion control treatment on academic emotions, motivation and achievement in an online mathematics course. Instructional Science, 40(1), 173-192. https://doi. org/10.1007/s11251-011-9165-6.

Larkin, K., y Jorgensen, R. (2015). 'I hate maths: Why do we need to do maths?' Using iPad video diaries to investigate attitudes and emotions towards mathematics in Year 3 and Year 6 students. International Journal of Science and Mathematics Education, 14(5), 1-20. doi: 10.1007/s10763-015-9621-x.

Leguizamón, J., Patiño, O., y Suárez, P. (2015). Tendencias didácticas de los docentes de matemáticas y sus concepciones sobre el papel de los medios educativos en el aula. Revista Educación Matemática, 27(3), 151-174.

Lewis, D. (2020). Student anxiety in standards-based grading in mathematics courses. Innovative Higher Education, 45(2), 153-164.

Luttenberger, S., Wimmer, S., y Paechter, M. (2018). Spotlight on math anxiety. Psychology Research and Behavior Management, 11, 311-322.

Mantooth, R., Usher, E. L., y Love, A. M. A. (2020). Changing classrooms bring new questions: Environmental influences, self-efficacy, and academic achievement. Learning Environments Research. Advance online publication. https://doi.org/10.1007/s10984020-09341-y.

Menekse, M., Anwar, S., y Purzer, S. (2018). Self-efficacy and mobile learning technologies: A case study of CourseMIRROR. En Self-Efficacy in instructional technology contexts (pp. 57-74). Cham: Springer. https:// doi.org/10.1007/978-3-319-99858-9_4.

Öçal, T. (2021). 'I remembered this mathematics course because...': How unforgettable mathematics experiences of pre-service early childhood teachers are related to their beliefs. International Journal of Mathematical Education in Science and Technology, 52(2), 1-17. https://doi.org/10.1080/0020739X.2020.1861349.

Orozco Santa María, A. M., García Ramírez, M. T., y Cepeda Villasana, L. A. (2019). Alfabetización digital desde un enfoque instrumental, cognitivo y emocional en estudiantes de turismo usando Blackboard. IE Revista de Investigación Educativa de la REDIECH, 10(19), 10(19), 11-35. https://doi.org/10.33010/ ie_rie_rediech.v10i19.300.

Osborne, J., Simon, S., y Collins, S. (2003). Attitudes towards science: A review of the literature and its implications. International Journal of Science Education, 25(9), 1049-1079.

Pizón, M., y Gallardo, A. (2000). Semántica versus sintaxis en la resolución de ecuaciones lineales. Educación Matemática, 12(2), 81-96.

Pyzdrowski, L., Sun, Y., Curtis, R., Miller, D., Winn, G., y Hensel, R. (2013). Readiness and attitudes as indicators for success in college calculus. International Journal of Science and Mathematics Education, 11(1), 529-554. doi: https://doi.org/10.1007/s10763-012-9352-1.

Radišiæ, J., Videnoviæ, M., y Baucal, A. (2015). Math anxiety - Contributing school and individual level factors. European Journal of Psychology of Education, 30(1), 1-20.

Rincón-Gallardo, S. (2019). Liberar el aprendizaje. El cambio educativo como movimiento social. México: Grano de Sal.

Rozgonjuk, D., Kraav, T., Mikkor, K., Orav-Puurand, K., y Täht, K. (2020). Ansiedad matemática entre estudiantes de STEM y ciencias sociales: los roles de la autoeficacia matemática y el enfoque profundo y superficial del aprendizaje. Revista Internacional de Educación STEM, 7(1), 1-11. 
Soto, J., Álvarez, A., Otero, G., Lampón, J., y Grinda, J. (2006). Educación media superior, Bacbillerato Intercultural. Guía del estudiante de la asignatura de Matemáticas Aplicadas I. Quinto semestre. México: Coordinación General de Educación Intercultural y Bilingüe de la Secretaría de Educación Pública.

Star, J. R., Chen, J. A., Taylor, M. W., Durkin, K., Dede, C., y Chao, T. (2014). Studying technology-based strategies for enhancing motivation in mathematics. International Journal of STEM Education, 1(1), 1-19.

Suárez-Pellicioni, M., Núñez-Peña, M. I., y Colomé, À. (2016). Math anxiety: A review of its cognitive consequences, psychophysiological correlates, and brain bases. Cognitive, Affective, \& Behavioral Neuroscience, 16(1), 3-22.

Tapia, M., y Marsh, G. E. (2004). An instrument to measure Mathematics attitudes. Academic Exchange Quarterly, 8(2), 16-21.

Tarango, J., Machin-Mastromatteo, J. D., y Romo-González, J. R. (2019). Evaluación según diseño y aprendizaje de Google Classroom y Chamilo. IE Revista de Investigación Educativa de la REDIECH, 10(19), 91-104.
Tezer, M., y Bozkurt, A. (2015). Determining attitudes and anxiety levels of students in need of protection towards Mathematics course. Procedia - Social and Behavioral Sciences (186), 269-273. https://doi. org/10.1016/j.sbspro.2015.04.197.

Thurm, D., y Barzel, B. (2020). Effects of a professional development program for teaching mathematics with technology on teachers' beliefs, self-efficacy and practices, ZDM, 52(7), 1411-1422.

Wu, C. H., Lin, W. T., y Shih, S. C. (2016). An exploratory study for evaluating mathematical anxiety in calculus e-assessment platform by using physiological signal analysis. En International Conference on Frontier Computing (pp. 435-441). Singapur: Springer.

Yeh, C. Y., Cheng, H. N., Chen, Z. H., Liao, C. C., y Chan, T. W. (2019). Enhancing achievement and interest in Mathematics learning through Math-Island. Research and Practice in Technology Enhanced Learning, 14(1), 1-19.

Zhai, X., y Shi, L. (2020). Understanding how the perceived usefulness of mobile technology impacts physics learning achievement: A pedagogical perspective. Journal of Science Education and Technology, 29(6), 743-757.

Cómo citar este artículo:

Dehesa De Gyves, N.., y López Ramírez, E. (2021). Ansiedad matemática, actitud y autoeficacia: un estudio sobre el efecto de $A p p$ Calc en estudiantes de ingeniería IE Revista de Investigación Educativa de la REDIECH, 12, e1229. doi: 10.33010/ie_rie_rediech.v12i0.1229. 